WHEAT

\section{Contested Parentage}

from a Correspondent

NEw interest in the evolutionary origins of the common wheat of agriculture, Triticum aestivum, has been stimulated by the discovery that some of its diploid relatives, in the genus Aegilops, contain genetic variation which affects meiotic chromosome pairing. $T$. aestivum is a hexaploid which contains the complete sets of chromosomes--known respectively as the A, B and D genomes - of three distinct diploid species. It has long been accepted that the A and D genomes were derived respectively from close ancestors of the contemporary diploids Triticum monococcum and Aegilops squarrosa. More uncertainty surrounds the origin of the B genome.

It should first be pointed out that in each genome of wheat the chromosomes relate genetically to corresponding, or homoeologous, chromosomes in each of the other genomes. Homoeologous chromosomes do not normally pair at meiosis, being prevented from doing so (Wall, Riley, Chapman and Gale, Genet. Res., 18, 311 and 329 ; 1971) by the activity of a single locus $(P h)$ which is distantly located on the long arm of chromosome 5B. The genotypes of speltoides and mutica have the capacity, in hybrids with $T$. aestivum, to suppress the activity of the $P h$ locus. Dover and Riley (Nature New Biology, 235, 61 ; 1972) and Kimber and Athwal (Proc. US Nat. Acad. Sci., 69, 912 ; 1972) have now described the existence of genetic variation which affects the occurrence of homoeologous meiotic pairing in hybrids between $T$. aestivum and $A e$. mutica and Ae. speltoides respectively.

Ae. speltoides, for reasons based on karyotype, gross plant morphology and geographical distribution, has been considered to be a strong candidate for recognition as the donor of the $B$ genome. Kimber and Athwal now interpret the patterns of meiotic chromosome pairing observed in wheatAe. speltoides combinations, in which the $P h$ activity is apparently not suppressed, as implying no closer relationship between wheat and Ae. speltoides than that between wheat and many other Aegilops species, so reducing the likelihood of Ae. speltoides being the source of the $B$ genome. The essence of Kimber and Athwal's argument is that, even if homoeologous pairing cannot occur, there should still be pairing between chromosomes of the $B$ genome and their full homologues in the $A e$. speltoides genome.

The strength of this case will ultimately depend on the cause of the lowpairing behaviour in the critical wheatAe. speltoides hybrids. It might be the result merely of a form of asynapsis, and this could be tested by making hybrids in which one wheat chromosome was disomic as a result of the cross $T$. aestivum tetrasomic for one chromosome $\times$ Ae. speltoides. If Kimber and Athwal are correct in their interpretation, the disomic chromosomes should pair normally, pairing being prevented only between homoeologues. Conclusions, based on other criteria, that Ae. speltoides was involved in the parentage of wheat would then be more deeply undermined.

Another question that might be resolved using the newly recognized pairing variants, as proposed by Dover and Riley, is the nature of the origin of the $P h$ allele of $T$. aestivum. Because no activity similar to that of $P h$ is known among the diploid relatives of wheat, it has long been thought that this allele must have arisen by mutation after chromosome 5B had been incorporated in polyploid wheat. The genotypes of Ae. speltoides and Ae. mutica that, in hybrids with wheat, result in low levels of meiotic pairing, may, however, carry alleles corresponding to that on chromosome 5B, in which case, as Dover and Riley suggest, there would be low pairing in similar hybrids lacking chromosome 5B because the presence of the diploid genome would compensate for its absence. This question, however, has still to be resolved.

The course of evolution of wheat is still a controversial issue. Clearly, difficulty is created by attempts to demonstrate parentage experimentally, with contemporary material, several thousands of generations of evolution subsequent to the hybridization event and there will always be uncertainty. Crop plant evolutionists now, however, have a better specification of the characteristics of the $B$ genome donor than formerly, when such bizarre proposals were made as that the $\mathrm{B}$ genome came from Agropyron triticeum, in spite of the fact that all the chromosomes of this species have subterminal centromeres and those of the B genome are median or submedian.

\section{VIROLOGY \\ Captive Genomes}

from our Cell Biology Correspondent

REOvirus places several claims for attention on virologists. For one thing, its genome is unusual; it comprises ten segments of double stranded RNA. For another, the virus particles contain an RNA transcriptase which, once the outer of the two capsids has been removed in vitro by proteolytic enzymes, will transcribe one of the two strands of each of the ten double stranded segments of the genome, all of which are themselves conserved.

These so-called sub-viral particles are an interesting source of messenger RNAs, and their properties at least raise the possibility that in vivo during the infectious cycle the reovirus genome, unlike the genomes of most

\title{
Selective Inhibition of RNA Synthesis
}

INFORMATION about new drugs or new ways to exploit tried and tested drugs, which allow experimentalists to probe further into the metabolic processes of eukaryotic cells, is always welcome and no doubt the suppliers of camptothecin can look forward to a boom in demand as a result of what Abelson and Penman have to say in Nature New Biology next Wednesday (May 31). They show that camptothecin, which is known to induce breakages in cellular DNA, also selectively interrupts the synthesis of high molecular weight RNAs in HeLa cell nuclei.

Abelson and Penman have confirmed that when HeLa cells, growing in suspension, are exposed to $1 \mu \mathrm{g} / \mathrm{ml}$. of the drug, DNA and RNA synthesis are both markedly inhibited whereas protein synthesis is hardly affected. Closer analysis of the small amount of RNA made in the presence of the drug reveals its selective effect. Although the average size of the molecules in ribosomal RNA precursor and heterogeneous nuclear RNA fractions is very much smaller than in controls the synthesis of 4 S RNA is only slightly impaired and 5S RNA synthesis seems to be completely unaffected by the drug at this and higher doses. Furthermore, whereas the truncated ribosomal RNA fractions made in the presence of the drug are rapidly degraded within the nucleus, the aberrant heterogeneous nuclear RNA is comparatively stable; it persists in the nucleus, being neither degraded nor exported to the cytoplasm.

How, at the molecular level, camptothecin brings about these changes remains to be elucidated; it is not apparently simply a matter of slowing down the rate of growth of RNA chains, and whether or not the drug inhibits RNA synthesis because it causes breaks in the template DNA is as yet an open question.

Abelson and Penman confirm one thing, however; camptothecin can be used to inhibit selectively synthesis of ribosomal and messenger RNAs without concomitantly blocking the synthesis of $4 \mathrm{~S}$ and $5 \mathrm{~S}$ RNAs or RNA synthesis in mitochondria. It will therefore increasingly find a place on the laboratory shelf. 
other viruses which are liberated from the virion in order that they may replicate, remains enclosed in a subviral particle similar if not identical to the sub-viral particles produced in vitro by exposing virions to proteolytic enzymes. That this is indeed the case has now been established by two groups ; Chang and Zweenik (Virology, 46, 544; 1971) and Silverstein et al. (ibid., 47, 797; 1972).

From the combined data obtained by these two groups and others the following picture of the reovirus infectious cycle emerges. The reovirus particle enters a cell in a phagocytic vacuole which promptly fuses with a lysosome within which the outer capsid of the virion is digested to leave a subviral particle. Analysis of the polypeptides in such subviral particles produced in vivo reveals that at least one polypeptide derived by cleavage from a protein in the outer capsid remains associated with the inner capsid, or core polypeptides enclosing the viral genome and that the in vivo sub-viral particles are not, therefore, identical to those produced in vitro. Nevertheless, like the in vitro sub-viral particles, those isolated from infected cells continue after extraction to synthesize single stranded RNA molecules which fall into three size classes, corresponding to the three classes of genomic RNA. Hybridization studies reveal, first, that the single stranded RNAs are the products of asymmetric transcription and, second, that among the total population of single stranded RNAs are transcripts of all ten segments of the genome.

In the infected cell the parental subviral particles seem to remain intact throughout the replication cycle and the single stranded RNAs are exported from the particles to the cytoplasm where they act as messengers. These single stranded RNAs also apparently act as templates for the replication of complementary chains to yield progeny genomes, and because little or no free double stranded RNA can be isolated from infected cells this replication presumably also occurs in a subviral particle. Finally, at late times in the infectious cycle newly made outer capsid proteins associate with the parental sub-viral particles as well as the progeny sub-viral particles. In short, as Silverstein et al. comment, sub-viral particles "are the structural and functional units of reovirus infection"; the infecting genome is never completely liberated from the structural proteins of the virion.

Like reovirus the syncytium forming or foamy viruses are also currently attracting considerable attention, but for very different reasons. These cytopathic and ubiquitous single stranded RNA viruses contain an RNA dependent DNA polymerase, or reverse transcriptase, similar to that present in the RNA tumour viruses. The mere presence of this enzyme in foamy virus particles suggests that their replication involves a DNA intermediate. Consonant with that idea, Parks et al. reported that actinomycin $\mathrm{D}$ greatly reduces the infectivity of simian foamy virus.

Now Parks and Todaro (ibid., 47, 673 ; 1972) have devised an 8 day plaque assay for bovine, feline and simian foamy viruses, have determined the growth cycle of simian foamy virus in human fibroblasts, and have shown that the thymidine analogue BudR at low concentrations markedly reduces the yield of infectious virus obtained when cells are infected with foamy viruses. This result also strongly implicates a DNA molecule in the replication of these viruses, which also share with the RNA tumour viruses a high resistance to inactivation by ultraviolet light.

The foamy viruses do not, however, seem to be oncogenic; all of the various cell types which Parks and Todaro have infected with one or other of these viruses have proved to be permissive for virus replication and have not assumed a transformed-cell phenotype. All of which goes to prove that the occurrence of reverse transcriptase in a single stranded RNA virus particle is not an infallible indication of an oncogenic potential.

\section{PROTEINS}

\section{Vistus in Viscosity}

from our Molecular Biology Correspondent

WITH only a capillary viscometer and a computer program or two, Harrington and Burke (Biochemistry, 11,1448 ; 1972) have deduced an explicit geometry for the myosin dimer, that exists in solution in rapid equilibrium with the monomer. This is more than a mere assertion of the enduring virtues of classical hydrodynamic method when the circumstances are favourable, for the myosin dimer is progressively favoured as the ionic strength decreases to the point at which filaments begin to form. It thus seems highly likely that it represents the first step in the process of thick-filament assembly. The structural evidence of Huxley indicates that the thick filaments contain parallel-packed myosin molecules, pointing in either direction from a central region in which the shafts meet, and there are no heads to form crossbridges.

Earlier work from the same laboratory having established that the solutions of myosin contain only the monomer and dimer in rapid equilibrium, Harrington and Burke have demonstrated that this is also the case for myosin rods, bereft of heads after papain treatment, and for light meromyosin, which is a proteolytically

\section{Origin of Mulfimeric QX174 DNA}

THE small genomes of many single stranded and double stranded DNA bacteriophages and viruses are covalently closed circular molecules. It has frequently been observed that when such genomes replicate in host cells a small proportion of the progeny molecules are twice as long as the parental molecules and even smaller proportions of the progeny are higher multiples of the parental genome.

There are two possible ways in which such multimeric DNA molecules might be generated-by recombination or by some failure to terminate replicationand evidence for one or other mechanism has been obtained by workers investigating the replication of various phages and viruses. Bacteriophage $\varphi \times 174$ is the most familiar of the coliphages with a circular single stranded DNA genome, and according to Benbow, Eisenberg and Sinsheimer's report in Nature New Biology next Wednesday (May 31) most circular multimeric $\varphi$ X174 DNA molecules arise not by recombination but as a result of some failure in the replication of the DNA.

Benbow et al. find, for example, that circular dimers and multimers of $\varphi$ X174 DNA are generated when the phage replicates in mutant Escherichia coli cells in which recombination is defective. Moreover, when a cell is coinfected with two mutant strains of $\varphi \mathrm{X} 174$, the DNA of which differs significantly in length, the small amount of circular dimeric and multimeric DNAs that arise fall into two classes, each class containing molecules that are exact multiples of the two parental DNA molecules.

Few if any of the multimeric circular molecules have lengths expected of molecules that comprise mixtures of the two parental DNAs. On the other hand, catenated molecules-forms in which two circular monomers are interlinked like the links of a chain-are generated in infected cells in which phage DNA replication is blocked. In doubly infected cells such catenated molecules comprise interlocked DNAs of the two parental strains.

In short, although Benbow et al. have no direct evidence about the precise molecular mechanisms involved, it seems clear that circular multimeric $\varphi$ X174 DNA molecules are generated by some failure in replication and not by a recombination event. As they caution, however, these findings pertain only to $\varphi \mathrm{X} 174$ and should not be extrapolated to other systems. 